\title{
Protective effect of nicorandil on hypoxia-induced apoptosis in HPAECs through inhibition of p38 MAPK phosphorylation
}

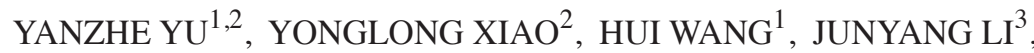 \\ XIANGRONG ZUO ${ }^{4}$, HONG WANG ${ }^{1}$ and WEIPING XIE ${ }^{1}$ \\ ${ }^{1}$ Department of Respiratory Medicine, The First Affiliated Hospital of Nanjing Medical University; \\ ${ }^{2}$ Department of Respiratory Medicine, Nanjing Drum Tower Hospital; Departments of ${ }^{3}$ Neurosurgery and ${ }^{4}$ ICU, \\ The First Affiliated Hospital of Nanjing Medical University, Nanjing 210029, P.R. China
}

Received September 14, 2012; Accepted November 30, 2012

DOI: $10.3892 / \mathrm{mmr} .2013 .1255$

\begin{abstract}
Endothelial cell apoptosis is induced under various conditions, including hypoxia, which is crucial for pulmonary hypertension. The present study aimed to investigate the protective effect of nicorandil against hypoxia-induced apoptosis in human pulmonary arterial endothelial cells (HPAEC) and the potential mechanisms involved in the regulation of p38 mitogen-activated protein kinase (MAPK). Following exposure to hypoxia for $24 \mathrm{~h}, 3$-(4,5-dimethylthiazol-2yl-)-2,5-diphenyltetrazolium bromide assay was performed to measure cell viability. Annexin V-propidium iodide double staining with flow cytometry and fluorescence staining of cells with Hoechst 33342 was then used to detect apoptosis. Expression of phosphorylated (phospho)-p38 MAPK, Bcl-2-associated X protein, B-cell lymphoma 2 , caspase- $8,-9$ and -3 and endothelial nitric oxide synthase (eNOS) was assayed by western blotting to investigate the mechanisms of action. Results showed that hypoxia significantly decreases cell viability by inducing cell apoptosis, while nicorandil and the p38 MAPK inhibitor, SB203580, reversed hypoxia-induced apoptosis. Nicorandil and SB203580 consistently inhibited the phosphorylation of p38 MAPK and expression of various apoptosis-related proteins induced by hypoxia. However, nicorandil increased the hypoxia-induced downregulation of eNOS expression and the effects of nicorandil were completely blocked by the mitochondrial ATP-sensitive potassium (mitoKATP) channel antagonist, 5-hydroxydecanoate. Nicorandil was identified to protect HPAEC from hypoxia-induced apoptosis by activating mitoKATP channels. The mechanisms by which this protective effect is mediated may involve inhibition of phospho-p38 MAPK and downstream cell death pathways.
\end{abstract}

Correspondence to: Dr Weiping Xie, Department of Respiratory Medicine, The First Affiliated Hospital of Nanjing Medical University, No. 300 Guangzhou Road, Nanjing 210029, P.R. China E-mail:wpxie@njmu.edu.cn

Key words: human pulmonary arterial endothelial cells, hypoxia, mitochondrial KATP channel, p38 MAPK

\section{Introduction}

Pulmonary hypertension $(\mathrm{PH})$ is induced under various conditions, including hypoxia and is defined as a hemodynamic abnormality caused by a number of structural and functional alterations. Exposure to hypoxia leads to sustained vascular contraction, persistent elevation in pulmonary artery pressure, vascular remodeling of the pulmonary arteries and eventually to right-side heart failure. Recent studies have confirmed that endothelial apoptosis is the initial and triggering event for $\mathrm{PH}(1)$. When exposed to various environmental triggers, including hypoxia, excessive apoptosis of pulmonary arterial endothelial cells (PAECs) leads to loss of pre-capillary arteriolar continuity, endothelial dysfunction and abnormal overgrowth of pulmonary arterial smooth muscle cells. These events may lead to increased pulmonary vascular resistance and appearance of abnormal apoptosis-resistant, hyperproliferative PAECs which form occlusive intimal and plexiform lesions (1). A number of studies have also reported the importance of endothelial nitric oxide synthase (eNOS) in the endothelial system. Endothelial dysfunction is characterized by reduced synthesis of NO (2-4) and hypoxia decreases expression of eNOS (5), which further jeopardizes endothelial function. Therefore, new treatments for $\mathrm{PH}$ should be directed towards antagonizing PAEC apoptosis and restoring eNOS expression.

p38 mitogen-activated protein kinases (p38 MAPKs) are a class of evolutionarily conserved serine/threonine protein kinases (6). Recent studies $(7,8)$ have focused on the response of p38 MAPK towards hypoxia and its role in the pathogenesis of hypoxic PH. Analysis of the cardiovascular and pulmonary vascular systems indicates that p38 MAPK is closely associated with endothelial function, particularly the regulation of endothelial cell proliferation/apoptosis and expression of eNOS (7,9-11).

Nicorandil is a mitochondrial ATP-sensitive potassium (mitoKATP) channel activator with a nitrate-like action. The molecule dilates peripheral and coronary arterioles and systemic veins by opening mitoKATP channels in vascular smooth muscle cells. Previous studies indicated that KATP channels may also affect the endothelin system. Nicorandil inhibits endothelial apoptosis, enhances eNOS expression and preserves endothelial function via activation of the mitoKATP 
channels in the endothelial cell (12-16). In addition, KATP channels have been reported to interact with various MAPK signals (3,17-19).

Therefore, we hypothesized that nicorandil antagonizes apoptosis induced by hypoxia and upregulates eNOS expression via activation of the mitoKATP channels in PAEC and this endothelial protective effect may involve the inhibition of p38 MAPK phosphorylation. To test this hypothesis, the effect of nicorandil on hypoxia-induced apoptosis in human PAEC (HPAEC), eNOS expression and phosphorylation of $\mathrm{p} 38$ MAPK was investigated.

\section{Materials and methods}

Materials. Cell culture medium components were purchased from Hyclone Laboratories (Logan, UT, USA). HPAECs were obtained from (ScienCell Research Laboratories, Carlsbad, CA, USA). SB203580, 3-(4,5-dimethylthiazol-2-yl)-2,5-diphenyltetrazolium bromide (MTT), 5-hydroxydecanoate (5-HD), Hoechst 33342, annexin V-fluoresceinisothocyanate (FITC) and propidium iodide (PI) were purchased from Sigma-Aldrich (St. Louis, MO, USA). Nicorandil was purchased from Tokyo Chemical Industry Co., Ltd. (Tokyo, Japan). Antibodies against phosphorylated (phospho)-p38 MAPK, p38 MAPK, Bcl-2-associated X protein (Bax), B-cell lymphoma 2, (Bcl-2), caspase- 8 and -9 , the cleaved form of caspase- 3 and eNOS were purchased from Cell Signaling Technology, Inc. (Beverly, MA, USA) and $\beta$-actin was obtained from Santa Cruz Biotechnology Inc., (Santa Cruz, CA, USA). AnaeroPack-Anaero was purchased from Mitsubishi Gas Chemical Co. (Tokyo, Japan).

Cell culture and treatment. HPAECs were plated in $100-\mathrm{mm}$ plastic tissue culture dishes and cultured in growth medium composed of $10 \%$ (v/v) FBS, 1\% (v/v) VEGF and $100 \mathrm{U} / \mathrm{ml}$ penicillin and streptomycin in a humidified incubator containing $5 \% \mathrm{CO}_{2}$ at $37^{\circ} \mathrm{C}$. Drugs and hypoxia treatments were performed when cells reached 70-80\% confluency. HPAECs were divided into 5 groups: i) control, cultured in normoxia $\left(20 \% \mathrm{O}_{2}, 5 \% \mathrm{CO}_{2}\right)$; ii) hypoxia alone, exposed to hypoxia for $24 \mathrm{~h}$; iii) hypoxia + nicorandil, treated with nicorandil $(10 \mu \mathrm{M}$, 100 and $1,000 \mu \mathrm{M}$ for MTT or $100 \mu \mathrm{M}$ for other methods) prior to $24 \mathrm{~h}$ hypoxia; iv) hypoxia + nicorandil $+5-\mathrm{HD}$, pretreated with 5-HD $(500 \mu \mathrm{M}) 30 \mathrm{~min}$ prior to nicorandil followed by hypoxia treatment; and v) hypoxia + SB203580, pretreated with SB203580 $(10 \mu \mathrm{M})$ prior to $24 \mathrm{~h}$ hypoxia. AnaeroPack-Anaero was used to create the hypoxic environment.

Cell growth assay. Cell viability of HPAEC was determined by measuring the MTT dye absorbance in cells. Cells were seeded in 96-well microtiter plates with $3 \times 10^{4}$ cells/well. Following drug treatment, the cells were exposed to hypoxia and MTT solution $(5 \mathrm{mg} / \mathrm{ml}$ in PBS) was added to the plates $(20 \mu \mathrm{l} /$ well $)$ and incubated for an additional $4 \mathrm{~h}$ at $37^{\circ} \mathrm{C}$. MTT solution was discarded and DMSO $(100 \mu \mathrm{l})$ was added to each well to solubilize formazan crystals formed in viable cells. A microplate reader was used to measure optical density at $570 \mathrm{~nm}$.

Flow cytometry. Cell apoptosis was measured by flow cytometry. HPAECs were seeded in 6-well plates (2x10 5 cells/well). Cells were treated as described above. Trypsin was added to each well following hypoxia and detached cells were collected and double stained for 10 min with FITC-coupled Annexin V protein and PI. Flow cytometry was performed with a 488-nm laser coupled to a cell sorter. Cells stained with Annexin V and PI or Annexin V only were considered necrotic and apoptotic, respectively.

Fluorescent staining of cells with Hoechst 33342. Cells were cultured in 24-well plates and treated at $\sim 80 \%$ confluency, as described above. Following hypoxia, the cells were washed with PBS prior to $20 \mathrm{~min}$ staining with Hoechst $33342(10 \mathrm{mg} / \mathrm{ml})$. Images of stained cells were captured by fluorescence microscopy and the percentage of apoptosis was calculated by counting ( $>500$ cells were scored/group).

Western blot analysis. Expression of phospho-p38 MAPK/p38 MAPK, Bax, Bcl-2, caspase-8 and -9 , the cleaved form of caspase-3 and eNOS was detected to study the mechanisms of nicorandil on apoptosis of HPAEC at the molecular level. Cell lysates contained 90\% RIPA buffer and 10\% PMSF and protease and phosphatase inhibitors were added to the cell culture dishes when the cells were $80 \%$ confluent. Lysates were collected and centrifuged at $14,000 \times \mathrm{g}$ for $15 \mathrm{~min}$ and supernatants were boiled with SDS loading buffer for $10 \mathrm{~min}$. Equal amounts of sample were loaded onto 12/15\% SDS-polyacrylamide gels and transferred to PVDF membranes (Millipore, Billerica, MA, USA). Membranes were blocked for $1 \mathrm{~h}$ in 5\% non-fat milk in PBS and then incubated with primary antibodies overnight at $4^{\circ} \mathrm{C}$. This process was followed by 3 TBST washes for $5 \mathrm{~min}$ and incubation with peroxidase-conjugated goat anti-rabbit IgG (1:10,000; Pierce Biotechnology, Inc., Rockford, IL, USA) for $1 \mathrm{~h}$ at room temperature. Membranes were again washed 3 times with TBST and developed using the ECL chemiluminescence detection system.

Statistical analysis. Data were expressed as the mean $\pm \mathrm{SE}$ and analyzed by one-way analysis of variance ANOVA followed by Tukey's post hoc test. $\mathrm{P}<0.05$ was considered to indicate a statistically significant difference.

\section{Results}

Effect of nicorandil on cell growth and death in hypoxia-treated HPAECs. HPAECs were treated with nicorandil, 5-HD or SB203580 in the presence or absence of hypoxia, as described. Following this, an MTT assay was performed to examine the effect of nicorandil on cell viability. Hypoxia treatment alone was found to significantly decrease cell growth (Fig. 1A), which was antagonized by nicorandil in a dose-dependent manner (Fig. 1B). The effect of nicorandil was completely blocked by the mitoKATP inhibitor, 5-HD (Fig. 1B). The p38 inhibitor SB203580 also had a similar effect to nicorandil on viability of HPAEC.

Nicorandil hypoxia-induced apoptosis in HPAEC. Apoptosis in HPAEC was detected by the Annexin V-FITC/PI double staining method (Fig. 2) to confirm the protective role of nicorandil against hypoxia-induced apoptosis. Frequency of apoptosis increased from $1.917 \pm 0.184$ in control to 

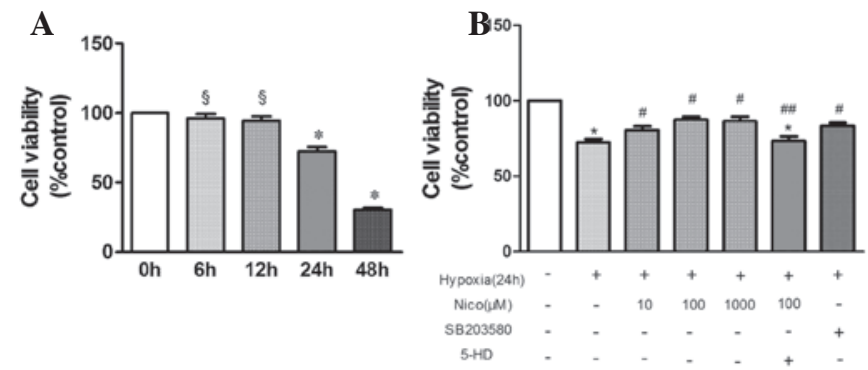

Figure 1. Cell viability using MTT assay. (A) Exposure to various durations of hypoxia. (B) Effect of various doses of nicorandil and SB203580 on HPAEC cell viability following exposure to hypoxia for $24 \mathrm{~h} .{ }^{8} \mathrm{P}>0.05$, vs. control; " $\mathrm{P}<0.05$, vs. control; ${ }^{\#} \mathrm{P}<0.05$, vs. hypoxia treatment alone; ${ }^{\# \#} \mathrm{P}<0.05$ vs. hypoxia + nicorandil. Results are shown as the mean $\pm \mathrm{SE}(\mathrm{n}=6)$. MTT, 3-(4,5-dimethylthiazol-2yl-)-2,5-diphenyltetrazolium bromide; HPAEC, human pulmonary arterial endothelial cells, Nico, nicorandil; 5-HD, 5-hydroxydecanoate.
A

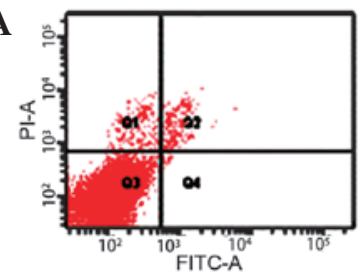

C

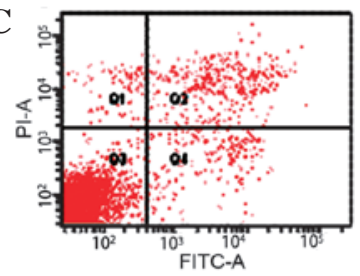

$\mathbf{E}$

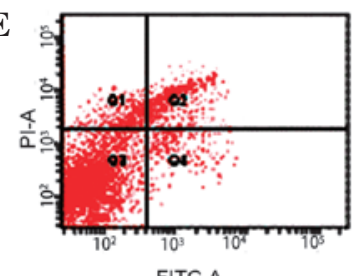

FITC-A
B

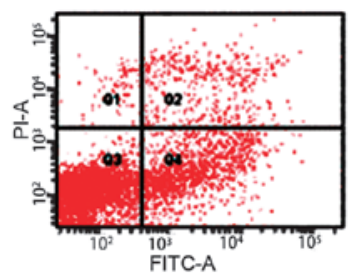

D

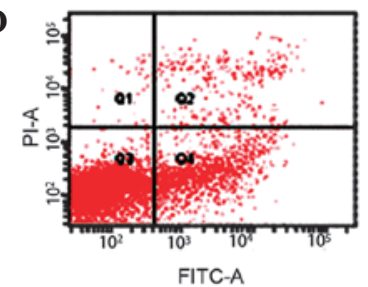

F

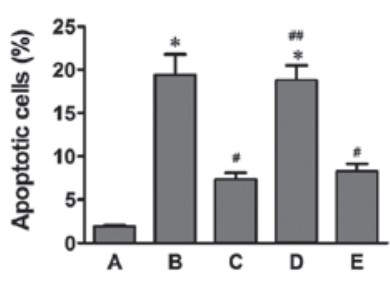

Figure 2. Apoptosis in HPAEC was detected by flow cytometry. Hypoxia for $24 \mathrm{~h}$ increased apoptosis in HPAEC. Nicorandil and SB203580 inhibited hypoxia-induced apoptosis and this protective effect was blunted by 5-HD. (A) Control; (B) hypoxia alone; (C) hypoxia + nicorandil; (D) hypoxia + nicorandil + 5-HD; (E) hypoxia + SB203580; and (F) quantification of flow cytometry. ${ }^{*} \mathrm{P}<0.05$, vs. control; ${ }^{*} \mathrm{P}<0.05$, vs. hypoxia treatment alone; and ${ }^{\# \#} \mathrm{P}<0.05$, vs. hypoxia + nicorandil. Results are shown as the mean \pm SE $(n=3)$. HPAECs, human pulmonary arterial endothelial cells; 5-HD, 5-hydroxydecanoate; FITC, fluoresceinisothocyanate.

$19.435 \pm 2.342 \%$ in the hypoxia group $(\mathrm{P}<0.05)$. However, treatment with nicorandil and SB203580 decreased apoptosis to $7.341 \pm 0.763$ and $8.312 \pm 0.851 \%$, respectively. No significant difference was found between pretreatment with 5-HD and hypoxia alone $(\mathrm{P}<0.05)$. Nicorandil was observed to protect HPAEC from hypoxia-induced apoptosis and this effect was blocked completely by 5-HD. The p38 MAPK inhibitor SB203580 demonstrated a similar protective effect.

To confirm the protective effect of nicorandil against hypoxia-induced apoptosis, Hoechst 33342 staining was
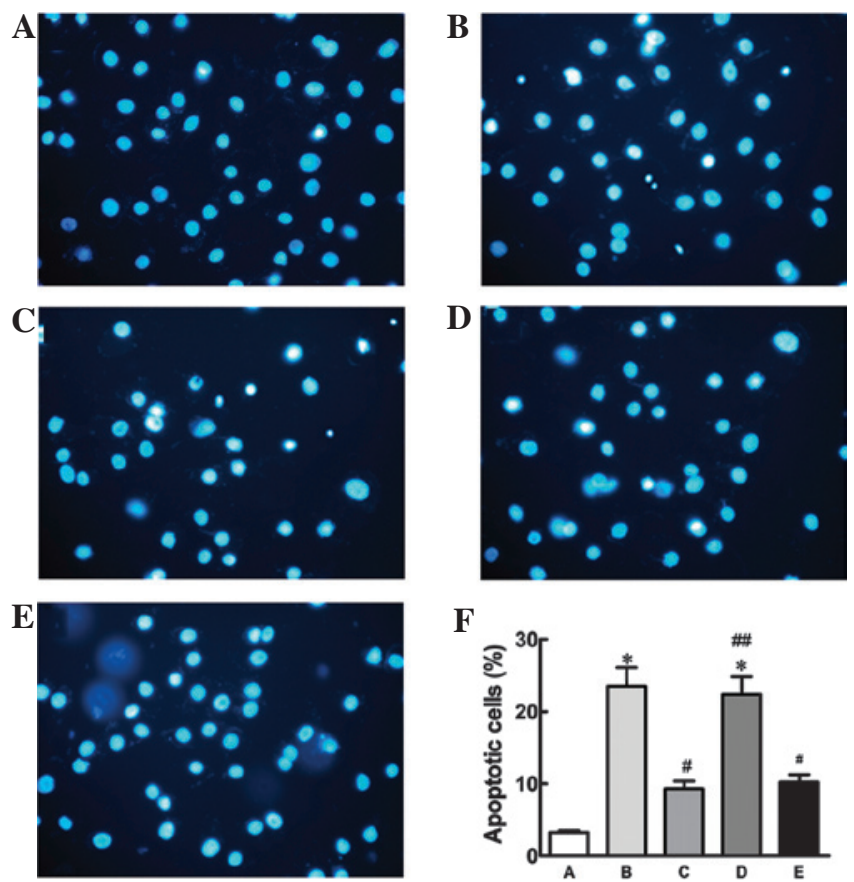

Figure 3. Apoptosis of HPAEC was analyzed by Hoechst 33342 staining (A) Control; (B) hypoxia alone; (C) hypoxia + nicorandil; (D) hypoxia + nicorandil + 5-HD; (E) hypoxia + SB203580; and (F) quantification of Hoechst 33342 staining. ${ }^{\#} \mathrm{P}<0.05$, vs. hypoxia treatment alone; and ${ }^{\# \#} \mathrm{P}<0.05$, vs. hypoxia + nicorandil. Results are shown as the mean \pm SE $(n>10)$. HPAECs, human pulmonary arterial endothelial cells; 5-HD, 5-hydroxydecanoate.

performed to detect apoptosis (Fig. 3). Compared with the control, hypoxia treatment alone increased apoptosis in cells, which was antagonized by nicorandil or SB203580. No significant difference was found between treatment with 5-HD prior to nicorandil and hypoxia alone, indicating that the protective effects of nicorandil against apoptosis were completely blocked by $5-\mathrm{HD}$.

Nicorandil inhibited phosphorylation of p38 induced by hypoxia. Results demonstrated that nicorandil protects HPEAC from hypoxia-induced apoptosis by opening mitoKATP channels. To investigate the effects of nicorandil on the phosphorylation of p38 MAPK induced by hypoxia, western blot analysis was used to determine expression levels of phospho- and total-p38 MAPK (Fig. 4A). The results demonstrated that hypoxia treatment alone increases phosphorylation of p38 MAPK. However, a significant decrease was observed in the nicorandil and SB20380 treatment groups, while 5-HD blocked the antagonistic effect of nicorandil against phosphorylation of p38 MAPK.

Nicorandil inhibited expression of apoptosis-related proteins induced by hypoxia. To further investigate the potential cell signaling mechanisms involved, apoptosis-related proteins Bax, Bcl2, caspase- 8 and -9 and cleaved caspase- 3 were assessed (Figs. 4B and 5). Expression of eNOS (Fig. 5) was also assayed. As revealed in Figs. 4 and 5, hypoxia alone increased the expression of these apoptotic proteins but reduced synthesis of eNOS. This effect was significantly antagonized by nicorandil and SB203580, while 5-HD completely blocked the beneficial effect of nicorandil. 
A
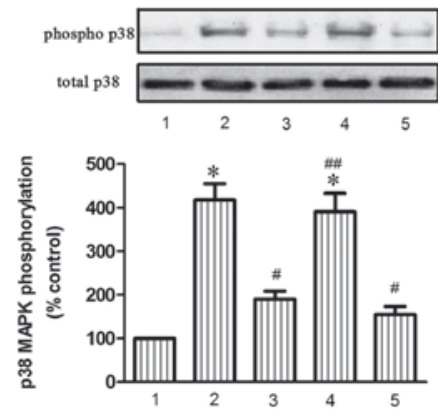

B

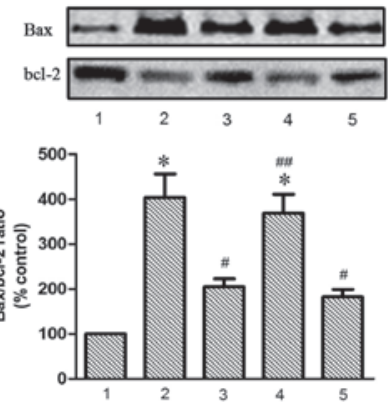

Figure 4. Nicorandil and SB203580 (A) inhibit phosphorylation of p38 MAPK and $(\mathrm{B})$ increase the $\mathrm{Bax} / \mathrm{Bcl}-2$ ratio induced in HPAEC following induction of hypoxia for 24 h. 1, control; 2, hypoxia alone; 3 , hypoxia + nicorandil 4 , hypoxia + nicorandil + 5-HD; and 5, hypoxia + SB203580. ${ }^{*} \mathrm{P}<0.05$, vs control; ${ }^{\#} \mathrm{P}<0.05$, vs. hypoxia treatment alone; ${ }^{\#} \mathrm{P}<0.05$, vs. hypoxia + nicorandil. Results are shown as the mean $\pm \mathrm{SE}(\mathrm{n}=4)$. HPAEC, human pulmonary arterial endothelial cells; Bax, Bcl-2-associated X protein; Bcl-2, B-cell lymphoma 2; 5-HD, 5-hydroxydecanoate; phospho, phosphorylated.

\section{A}
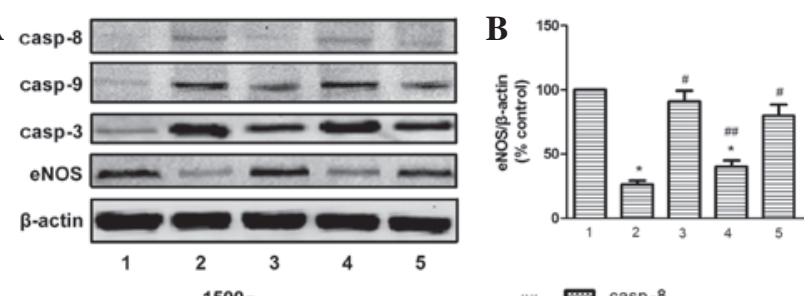

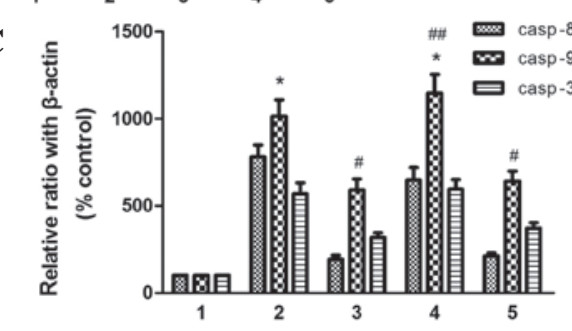

Figure 5. Effect of nicorandil and SB203580 on casp-8, -9 and -3 and eNOS in HPAEC following exposure to hypoxia for $24 \mathrm{~h}$. 1, control; 2, hypoxia alone; 3 , hypoxia + nicorandil; 4, hypoxia + nicorandil + 5-HD; 5, hypoxia + SB203580 ${ }^{*} \mathrm{P}<0.05$, vs. control; ${ }^{\#} \mathrm{P}<0.05$, vs. hypoxia treatment alone; ${ }^{\# \#} \mathrm{P}<0.05$, vs. hypoxia + nicorandil. Results are thrown as the mean $\pm \mathrm{SE}(\mathrm{n}=4)$. Casp, caspase; eNOS, endothelial nitric oxide synthase; HPAEC, human pulmonary arterial endothelial cells; 5-HD, 5-hydroxydecanoate.

\section{Discussion}

Results of the present study support our hypothesis that nicorandil protects endothelial cell function by opening mitoKATP channels and reversing activation of p38 MAPK. A number of studies have reported a protective role of nicorandil against cell apoptosis in various cell types. In the cardiovascular system, nicorandil appears to reduce mitochondrial impairment and inhibit apoptosis in cardiomyocytes by various mechanisms $(12,13,19,20)$. Similar results were also found in studies involving neurons (21). In vascular endothelial cells, nicorandil inhibits serum starvation-induced apoptosis (22). In the present study, the effects of nicorandil on hypoxia-induced apoptosis in HPAEC were evaluated. Results demonstrate that nicorandil maintained cell viability in a dose-dependent manner (Fig. 1B) by antagonizing hypoxia-induced apoptosis (Figs. 2 and 3). This maintenance was completely abrogated by treatment with 5-HD, a mitoKATP channel inhibitor, indicating that this effect was mediated by mitoKATP channels in HPAEC.

Several studies have revealed a correlation between nicorandil and the MAPK signaling pathway (17-19,23), particularly p38 MAPK, a kinase associated with apoptosis $(11,24,25)$. Previously, hypoxia was demonstrated to induce apoptosis of HUVECs in an in vitro capillary model (24) by activating p38 MAPK. In an additional study (11), inhibition of p38 MAPK reduced pyrogallol-induced apoptosis. In this study, the p38 MAPK inhibitor, SB203580, markedly reduced hypoxia-induced apoptosis in HPAEC (Figs. 2 and 3). Results indicate that p38 MAPK is a major mediator of hypoxia-induced apoptosis. Therefore, we suggest that the effect of nicorandil against endothelial apoptosis may involve the regulation of p38 MAPK. To test this hypothesis, the effect of nicorandil on phosphorylated p38 MAPK was analyzed. Hypoxia increased expression of phospho-p38, consistent with previous studies $(7,8)$. Nicorandil and SB203580 markedly decreased phosphorylation of p38 MAPK, while this effect was reversed by 5-HD. Results indicate that nicorandil inhibits phosphorylation of p38 MAPK via activation of the mitoKATP channels. The association between mitoKATP channels and p38 MAPK remains to be adequately studied, particularly in PAEC. In an in vivo cardiac model, activation of mitoKATP channels restored protection of preconditioning via p38 MAPK (17), while an additional study using microglia cells revealed that activation of microglial KATP channels inhibits rotenone-induced neuroinflammation by deactivating p38 MAPK (18). Results of the present study confirm this interaction between mitoKATP channels and p38 MAPK in PAEC when exposed to a hypoxic environment.

The cell death signals involved in the protective effect of nicorandil against hypoxia-induced apoptosis were also explored. We found that nicorandil and SB203580 reduced the $\mathrm{Bax} / \mathrm{Bcl} 2$ ratio and downregulated the expression of caspase-8, -9 and -3 , compared with hypoxia alone. The effect of nicorandil on these proteins was completely eliminated by 5 -HD. Cell apoptosis is induced by the mitochondrial and death receptor signaling pathways. The former involves translocation of Bax, leading to caspase-9 activation via the release of cytochrome c. The latter is triggered by members of the death receptor family followed by recruitment of caspase- 8 . Caspase- 8 and -9 activation also activates caspase-3, eventually leading to cell apoptosis. Early studies involving various cell types demonstrated that nicorandil is a mitoKATP channel activator and largely affects the mitochondrial pathway by reducing the $\mathrm{Bax} / \mathrm{Bcl} 2$ ratio and caspase-3 activation (13). Present results indicate that nicorandil also affects the death receptor pathway. It is well known that activation of p38 MAPK is a critical event leading to induction of the cell death pathway $(26,27)$. Consistent with these observations, the present study confirmed that nicorandil downregulates 
the two distinct cell death signaling pathways via deactivation of p38 MAPK (Fig. 5).

A number of studies (14-16) have demonstrated the critical role of eNOS in PH, particularly in the maintenance of endothelial function. Findings of studies on cardiovascular disease have shown that nicorandil protects endothelial function (14) and enhances eNOS expression (16). In a monocrotaline-induced pulmonary arterial hypertension model, similar effects of nicorandil were identified (15). Therefore, in the present study, expression of eNOS was determined and regulatory mechanisms were explored. Results indicate that nicorandil induced significant upregulation of eNOS, which was suppressed by hypoxia via deactivation of p38 MAPK (Figs. 4 and 5). These results are in accordance with a previous study (9) on the effects of p38 MAPK on eNOS, which indicated that activation of p38 MAPK is responsible for the downregulation of eNOS.

In previous years, various cell signaling pathways involved in hypoxia-induced apoptosis have been extensively studied in various cell types $(22,28,29)$. Additional studies are required to determine whether the beneficial effect of nicorandil against apoptosis and endothelial dysfunction induced by hypoxia also involves other signaling pathways. Future studies are likely to focus on the mechanism by which nicorandil inhibits phosphorylation of p38 MAPK and also on the involvement of additional cell signaling pathways, including phosphoinositide 3-kinase/AKT, c-Jun N-terminal kinase, extracellular signal-regulated kinase $1 / 2$ and nuclear factor- $\kappa \mathrm{B}$ and the cross-talk between them.

In conclusion, results of this study have demonstrated that nicorandil protects HPAEC from hypoxia-induced apoptosis by inhibiting the mitochondrial and death receptor pathways and aids maintenance of endothelial function by restoring eNOS expression. This protective effect is hypothesized to be associated with deactivation of p38 MAPK via mitoKATP channels.

\section{Acknowledgements}

The present study was supported by NSFC (no. 81273571).

\section{References}

1. Jurasz P, Courtman D, Babaie S and Stewart DJ: Role of apoptosis in pulmonary hypertension: from experimental models to clinical trials. Pharmacol Ther 126: 1-8, 2010.

2. Linke A, Recchia F, Zhang X and Hintze TH: Acute and chronic endothelial dysfunction: implications for the development of heart failure. Heart Fail Rev 8: 87-97, 2003.

3. Kawashima S and Yokoyama M: Dysfunction of endothelial nitric oxide synthase and atherosclerosis. Arterioscler Thromb Vasc Biol 24: 998-1005, 2004.

4. Triggle CR, Hollenberg M, Anderson TJ, et al: The endothelium in health and disease - a target for therapeutic intervention. J Smooth Muscle Res 39: 249-267, 2003.

5. Steudel W, Scherrer-Crosbie M, Bloch KD, et al: Sustained pulmonary hypertension and right ventricular hypertrophy after chronic hypoxia in mice with congenital deficiency of nitric oxide synthase 3. J Clin Invest 101: 2468-2477, 1998.

6. Cuadrado A and Nebreda AR: Mechanisms and functions of p38 MAPK signalling. Biochem J 429: 403-417, 2010.

7. Weerackody RP, Welsh DJ, Wadsworth RM and Peacock AJ: Inhibition of p38 MAPK reverses hypoxia-induced pulmonary artery endothelial dysfunction. Am J Physiol Heart Circ Physiol 296: H1312-H1320, 2009.

8. Zhang CL, Song F, Zhang J and Song QH: Hypoxia-induced Bcl-2 expression in endothelial cells via p38 MAPK pathway. Biochem Biophys Res Commun 394: 976-980, 2010.
9. Xing F, Jiang Y, Liu J, et al: Downregulation of human endothelial nitric oxide synthase promoter activity by p38 mitogen-activated protein kinase activation. Biochem Cell Biol 84: 780-788, 2006.

10. Grossini E, Molinari C, Caimmi PP, Uberti F and Vacca G: Levosimendan induces NO production through p38 MAPK, ERK and Akt in porcine coronary endothelial cells: role for mitochondrial K(ATP) channel. Br J Pharmacol 156: 250-261, 2009.

11. Han YH, Moon HJ, You BR, Kim SZ, Kim SH and Park WH: JNK and p38 inhibitors increase and decrease apoptosis, respectively, in pyrogallol-treated calf pulmonary arterial endothelial cells. Int J Mol Med 24: 717-722, 2009.

12. Sanbe A, Marunouchi T, Yamauchi J, Tanonaka K, Nishigori H and Tanoue A: Cardioprotective effect of nicorandil, a mitochondrial ATP-sensitive potassium channel opener, prolongs survival in HSPB5 R120G transgenic mice. PLoS One 6: e18922, 2011.

13. Nishikawa S, Tatsumi T, Shiraishi J, et al: Nicorandil regulates Bcl-2 family proteins and protects cardiac myocytes against hypoxia-induced apoptosis. J Mol Cell Cardiol 40: 510-519, 2006.

14. Zhao JL, Yang YJ, Chen JL, Kang LM, Wu Y and Gao RL: Nicorandil reduces myocardial no-reflow by protection of endothelial function via the activation of KATP channel. Clin Chim Acta 374: 100-105, 2006.

15. Hongo M, Mawatari E, Sakai A, et al: Effects of nicorandil on monocrotaline-induced pulmonary arterial hypertension in rats. J Cardiovasc Pharmacol 46: 452-458, 2005.

16. Horinaka S, Kobayashi N, Higashi T, Hara K, Hara S and Matsuoka H: Nicorandil enhances cardiac endothelial nitric oxide synthase expression via activation of adenosine triphosphate-sensitive $\mathrm{K}$ channel in rat. J Cardiovasc Pharmacol 38: 200-210, 2001

17. Iliodromitis EK, Aggeli IK, Gaitanaki C, et al: p38-MAPK is involved in restoration of the lost protection of preconditioning by nicorandil in vivo. Eur J Pharmacol 579: 289-297, 2008.

18. Zhou F, Yao HH, Wu JY, Ding JH, Sun T and Hu G: Opening of microglial K(ATP) channels inhibits rotenone-induced neuroinflammation. J Cell Mol Med 12: 1559-1570, 2008.

19. Nagata K, Obata K, Odashima M, et al: Nicorandil inhibits oxidative stress-induced apoptosis in cardiac myocytes through activation of mitochondrial ATP-sensitive potassium channels and a nitrate-like effect. J Mol Cell Cardiol 35: 1505-1512, 2003.

20. Akao M, Teshima Y and Marban E: Antiapoptotic effect of nicorandil mediated by mitochondrial atp-sensitive potassium channels in cultured cardiac myocytes. J Am Coll Cardiol 40: 803-810, 2002.

21. Teshima Y, Akao M, Baumgartner WA and Marban E: Nicorandil prevents oxidative stress-induced apoptosis in neurons by activating mitochondrial ATP-sensitive potassium channels. Brain Res 990: 45-50, 2003.

22. Date T, Taniguchi I, Inada K, et al: Nicorandil inhibits serum starvation-induced apoptosis in vascular endothelial cells. J Cardiovasc Pharmacol 46: 721-726, 2005.

23. Chao HH, Hong HJ, Sung LC, Chen JJ, Cheng TH and Liu JC: Nicorandil attenuates cyclic strain-induced endothelin-1 expression via the induction of activating transcription factor 3 in human umbilical vein endothelial cells. Eur J Pharmacol 667: 292-297, 2011.

24. Eguchi R, Suzuki A, Miyakaze S, Kaji K and Ohta T: Hypoxia induces apoptosis of HUVECs in an in vitro capillary model by activating proapoptotic signal p38 through suppression of ERK1/2. Cell Signal 19: 1121-1131, 2007.

25. Hartel FV, Holl M, Arshad M, et al: Transient hypoxia induces ERK-dependent anti-apoptotic cell survival in endothelial cells. Am J Physiol Cell Physiol 298: C1501-C1509, 2010.

26. Park MT, Choi JA, Kim MJ, et al: Suppression of extracellular signal-related kinase and activation of p38 MAPK are two critical events leading to caspase-8- and mitochondria-mediated cell death in phytosphingosine-treated human cancer cells. J Biol Chem 278: 50624-50634, 2003.

27. Lin FL, Hsu JL, Chou CH, Wu WJ, Chang CI and Liu HJ: Activation of p38 MAPK by damnacanthal mediates apoptosis in SKHep 1 cells through the DR5/TRAIL and TNFR1/TNF-alpha and p53 pathways. Eur J Pharmacol 650: 120-129, 2011.

28. Ikeda R, Che XF, Ushiyama M, et al: 2-Deoxy-D-ribose inhibits hypoxia-induced apoptosis by suppressing the phosphorylation of p38 MAPK. Biochem Biophys Res Commun 342: 280-285, 2006.

29. Liou SF, Ke HJ, Hsu JH, et al: San-huang-xie-xin-tang prevents rat hearts from ischemia/reperfusion-induced apoptosis through eNOS and MAPK pathways. Evid Based Complement Alternat Med: 915051, 2011. 